\title{
Metastable Liquid-Liquid and Solid-Liquid Phase Boundaries in Polymer-Solvent-Nonsolvent Systems
}

\author{
P. VAN DE WITTE, * P. J. DIJKSTRA, J. W. A. VAN DEN BERG, J. FEIJEN \\ Department of Chemical Technology and Institute for Biomedical Technology, University of Twente, P. O. Box 217, \\ 7500 AE Enschede, The Netherlands
}

Received 21 November 1995; revised 31 July 1996; accepted 20 September 1996

\begin{abstract}
In general liquid-liquid demixing processes are responsible for the porous morphology of membranes obtained by immersion precipitation. For rapidly crystallizing polymers, solid-liquid demixing processes also generate porous morphologies. In this study, the interference of both phase transitions has been analyzed theoretically using the Flory-Huggins theory for ternary polymer solutions. It is demonstrated that four main thermodynamic and kinetic parameters are important for the structure formation in solution: the thermodynamic driving force for crystallization, the ratio of the molar volumes of the solvent and the nonsolvent, the polymer-solvent interaction parameter, and the rate of crystallization of the polymer compared to the rate of solvent-nonsolvent exchange. An analysis of the relevance of each of these parameters for the membrane morphology is presented. (C) 1997 John Wiley \& Sons, Inc. J Polym Sci B: Polym Phys 35: 763-770, 1997

Keywords: melting transitions; liquid-liquid demixing; immersion precipitation; membranes; nonequilibrium phenomena
\end{abstract}

\section{INTRODUCTION}

During the past two decades porous membranes have been developed for a wide variety of applications. ${ }^{1,2}$ For each of these applications the morphology of the membrane has to be optimized. A large amount of research has been performed to understand the fundamentals of the membrane formation by controlled phase separation of polymer solutions. ${ }^{3-26}$ At least three types of phase transitions can play an important role in the structure formation and fixation: (1) liquid-liquid demixing, (2) solidliquid demixing, and (3) the glass transition. Combinations of liquid-liquid demixing and the glass transition or solid-liquid demixing are held responsible for the porous morphology

* Present address: Philips Research Laboratories, Prof. Holstlaan 4, 5656 AA, Eindhoven, The Netherlands Correspondence to: J. Feijen

(c) 1997 John Wiley \& Sons, Inc. CCC 0887-6266/97/050763-08 of the membranes. For thermally induced phase separation a good picture of the interference of phase transitions processes is now available. For ternary systems, where the immersion of a polymer solution in an excess of nonsolvent induces the phase transitions, fewer studies have been performed. In the present article a more detailed thermodynamical analysis of the competition between liquid-liquid demixing and solid-liquid demixing for ternary systems is presented. The findings will be discussed in relation to the structure generation in the polymer solution during immersion precipitation.

\section{THEORY}

Here the discussion of interference of phase transitions is based on the Flory-Huggins theory. ${ }^{27-29}$ Physically more meaningful thermodynamic theories than the Flory-Huggins theory are currently 
available (e.g., equation of state theories). However, because of its simplicity the Flory-Huggins theory is still suitable to obtain a general understanding of these complex systems. Furthermore the Flory-Huggins theory can provide qualitative and easily applicable rules for the choice of the components of the membrane forming combination.

The general Flory-Huggins description for the Gibbs free energy of mixing of three component systems was derived by Pouchly et al. [eq. (1) $]:^{30,31}$

$$
\begin{gathered}
\frac{\Delta G_{M}}{R T}=n_{1} \ln \phi_{1}+n_{2} \ln \phi_{2}+n_{3} \ln \phi_{3}+g_{12} n_{1} \phi_{2} \\
+g_{13} n_{1} \phi_{3}+g_{23} n_{2} \phi_{3}+g_{T} n_{1} \phi_{2} \phi_{3} .
\end{gathered}
$$

$n_{i}$ represents the number of moles of component $i$ ( $i=1$ : nonsolvent; $i=2$ : solvent; $i=3$ : polymer ). $\phi_{i}$ represents the volume fraction of component $i$ and the $g_{i j}$ parameters represent the binary interaction parameters between the components $i$ and $j . g_{T}$ is a ternary interaction parameter and corrects empirically for the application of binary interaction parameters to ternary systems. It should be kept in mind that all interaction parameters $g_{i j}$ can depend on the ratio $\phi_{i} / \phi_{j}$ and that $g_{T}$ is known to depend on both $\phi_{1} / \phi_{2}$ and $\phi_{3} .{ }^{31}$

In a first approximation we assume the interaction parameters to be constants: the $g_{i j}$ equal the Flory-Huggins $\chi_{i j}$. In addition, we neglect the influence of molecular weight distributions and of the ternary interaction parameter $g_{T}$. The resulting equation still contains the general thermodynamic characteristics of ternary systems, but the calculations are simplified to a great extent.

From eq. (1) equations can be derived which describe the binodal, the critical point, and the spinodal for a ternary system. ${ }^{14,16,29}$ Routes to solve the equations are presented very clearly by Yilmaz and $\mathrm{McHugh}{ }^{16}$ and Altena et al. ${ }^{14}$ and will not be repeated here. The derivation of the equation for the solid-liquid miscibility gap for ternary systems proceeds in a similar way as the derivation of the equation for binary systems. ${ }^{13}$ The result is eq. (2):

$$
\begin{aligned}
& \frac{1}{T_{m}}-\frac{1}{T_{m}^{0}}=-\frac{R}{\Delta H_{m}^{0}} \frac{v_{u}}{v_{1}} \\
& \quad \times\left\{r \ln \phi_{3}+r\left(1-\phi_{3}\right)-\phi_{1}-s \phi_{2}\right. \\
& \left.\quad+\left(\chi_{13} \phi_{1}+s \chi_{23} \phi_{2}\right)\left(\phi_{1}+\phi_{2}\right)-\chi_{12} \phi_{1} \phi_{2}\right\} .
\end{aligned}
$$

$T_{m}$ and $T_{m}^{0}$ are the melting temperature of the polymer in solution and the hypothetical equilibrium melting temperature of the polymer in the solid state, respectively. $v_{u}$ indicates the molar volume of a repeat unit of the polymer and $\Delta H_{m}^{0}$ is the heat of fusion for $100 \%$ crystalline polymer. The $s$ and $r$ are molar volume ratios $\left(s=v_{1} / v_{2}\right.$ and $r=v_{1} / v_{3}, v_{i}=$ molar volume of component $i$ ). The $v_{1}$, and the variables that represent the properties of the semicrystalline polymer, can be grouped into a dimensionless parameter $A$. This parameter can be regarded as a measure for the thermodynamic driving force for crystallization of the polymer:

$$
A=-\left(\frac{1}{T_{m}}-\frac{1}{T_{m}^{0}}\right)\left(\frac{\Delta H_{m}^{0}}{R}\right)\left(\frac{v_{1}}{v_{u}}\right) .
$$

The thermodynamic parameters that are important for the phase transitions are (1) the thermodynamic interaction parameters, (2) the molar volumes, and ( 3 ) the dimensionless parameter $A$. The influence of each of these parameters will be discussed in detail.

\section{RESULTS}

As already mentioned, the influence of the interaction parameters on the location of the binodal, the spinodal, and the critical point have been discussed by others., ${ }^{3,15,23}$ Our results agree completely with the results obtained by these authors. The trends on the influence of the variables in the equations on the position of the liquid-liquid miscibility gap in the phase diagram can be summarized as follows:

- The polymer-nonsolvent interaction parameter $\left(\chi_{13}\right)$ determines to a great extent the surface area of the liquid-liquid miscibility gap. High values for the polymer-nonsolvent interaction parameters also imply that the point of intersection of the liquid-liquid miscibility gap with the polymer-nonsolvent axis is located at very high polymer concentrations.

- Solvents and nonsolvents with high mutual affinities ( low $\chi_{12}$ ) strongly increase the magnitude of the liquid-liquid miscibility gap.

- Low compatibility of the solvent-nonsolvent mixture (high $\chi_{12}$ ) results in large differ- 


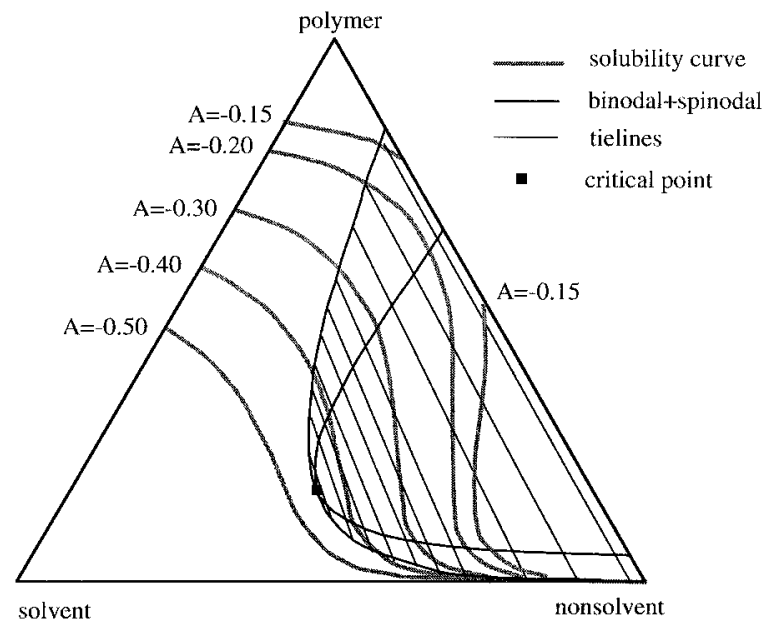

Figure 1. Calculated phase diagrams for several values of A. Parameters $s=1 ; r=0.1 ; \chi_{13}=1.5 ; \chi_{23}$ $=0.2 ; \chi_{12}=0.5$.

ences in solvent/nonsolvent ratio between the equilibrium phases.

- Polymers and solvents with high mutual affinity (low $\chi_{23}$ ) decrease the magnitude of the liquid-liquid miscibility gap, especially at low values of $\chi_{12}$.

- In a first approximation (minor) changes in molecular weight, molecular weight distribution, and molar volume are negligible.

When solid-liquid demixing is included the equilibrium phase diagram becomes more complex. For the construction of equilibrium phase diagrams several requirements have to be fulfilled. A general discussion on these requirements is presented by Koningsveld et al. ${ }^{32}$ In Figure 1 the phase diagram of a polymer in a solvent/nonsolvent mixture is presented. Both the liquid-liquid miscibility gap and the solubility curve (the border of the solid-liquid miscibility gap) are shown independently. The solubility curve is indicated for varying values of $A$.

The solubility curve starts at relatively high polymer concentrations at the polymer-solvent axis. Due to the lower average solvent quality of the solvent/nonsolvent mixture the transition shifts to lower polymer concentrations at increasing nonsolvent/solvent ratios. In a way, similar to the binary cases, the solid-liquid transition makes a Van der Waals-like loop in the liquidliquid miscibility gap due to the higher free energy of mixing of the homogeneous three-component solution in this composition range. Like in binary systems, the extremes of the solubility curve seem to be located on the spinodal. ${ }^{4,33}$ The part of the solubility curve that is situated in the liquid-liquid miscibility gap is metastable or unstable and does not have significance under equilibrium conditions.

At the entrance point of the solubility curve in the liquid-liquid miscibility gap the crystalline polymer is in equilibrium with a concentrated polymer solution. The concentrated liquid phase is also in equilibrium with a dilute polymer solution. Therefore, the solubility curve has to intersect the binodal exactly at the other end of the tie line at the intersection point. The equilibrium construction for this phase diagram is presented in Figure 2. At high polymer concentrations the crystalline polymer is in equilibrium with a liquid phase. At low polymer concentrations and low nonsolvent concentrations two liquid phases are in equilibrium. At high nonsolvent concentrations also a solid-liquid equilibrium exists. The two solid-liquid equilibria and the liquid-liquid equilibrium are separated by a three-phase region. These phase diagrams obey the thermodynamic criteria for phase diagrams established in ref. 32.

For increasing (i.e., less negative) $A$ values the solid-liquid miscibility gap becomes smaller. The $\mathrm{s}$ shape of the loop within the liquid-liquid miscibility gap becomes more prominent. For $A$ $=-0.15$ a part of the curve lies outside of the

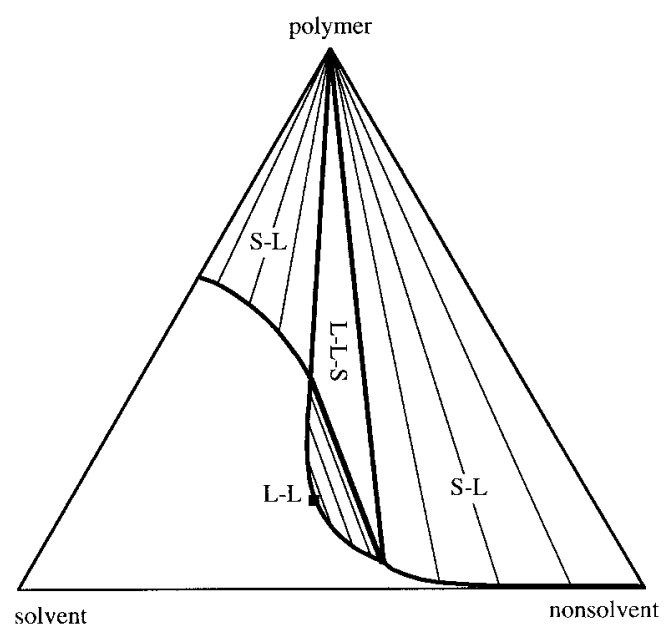

Figure 2. Equilibrium construction of a phase diagram exhibiting both a liquid-liquid and a solid-liquid miscibility gap (see Fig. 1). Two solid-liquid equilibria are indicated (S-L), one liquid-liquid equilibrium ( L$\mathrm{L}$ ), and one three phase equilibrium (L-L-S). Parameters $s=1 ; r=0.1 ; \chi_{13}=1.5 ; \chi_{23}=0.2 ; \chi_{12}=0.5 ; A$ $=-0.40$. 


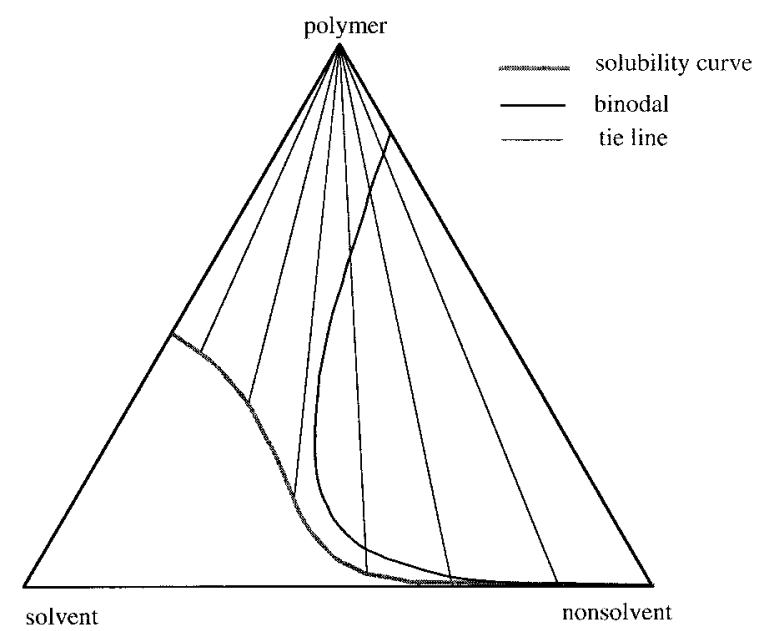

Figure 3. Equilibrium phase diagram of Figure 1 for $A=-0.50$. The position of the binodal is also indicated but this curve does not represent an equilibrium transition. Parameters $s=1 ; r=0.1 ; \chi_{13}=1.5 ; \chi_{23}=0.2$; $\chi_{12}=0.5$.

triangle. When the solubility curve curve does not intersect with the binodal at high polymer concentrations the solid-liquid and the liquid-liquid miscibility gap are completely separated. However, still branches of the solubility curve can exist in the liquid-liquid miscibility gap at lower polymer concentrations. These branches do not cross the binodal anymore but are situated completely in the metastable areas and the unstable areas of the liquid-liquid miscibility gap.

For low values of $A$ the solubility curve is located at lower nonsolvent concentrations than the liquid-liquid miscibility gap over the entire composition range. The equilibrium construction of the phase diagram for $A=-0.50$ is presented in Figure 3. For $A=-0.50$ liquid-liquid demixing does not have significance under equilibrium conditions.

\section{Influence of Interaction Parameters on the Phase Diagrams}

The effect of the interaction parameters on the size of the liquid-liquid miscibility gap has been presented earlier. From eqs. (1) and (2) it is clear that increases in $\chi_{23}$ and $\chi_{13}$ and decreases in $\chi_{12}$ will promote solid-liquid demixing. However, the influence of the parameters on the size of the liquid-liquid miscibility gap proceeds in a similar way. It is difficult to assess beforehand the relative importance of changes in the values of the interaction parameters for both transitions. The point of intersection of the solubility curve with the polymer-rich branch of the binodal appears to be suitable as a basis for the discussion. The point of intersection can be calculated by simultaneously solving the equations for the liquid-liquid miscibility gap and the solid-liquid miscibility gap. From calculations it appears that the influence of the interaction parameters on the polymer concentration at the intersection point at high values of $A$ is rather small (data not shown). Higher values of $\chi_{13}$ tend to increase the polymer concentration at the point of intersection except at high $\chi_{12}$ values. In most of the cases, low $\chi_{12}$ values decrease the polymer concentration at the point of intersection.

The effect of the value of the polymer-solvent interaction parameter is much larger than the effects of changes in the values for the other interaction parameters especially at low values for $A$. In Figure 4 the transitions are indicated for two sets of interaction parameters with different values for $\chi_{23}$.

\section{Influence of Ratios of Molar Volumes ( $s$ and $r$ )}

Variations in $r$ are of minor influence on the location of the solubility curve and the liquid-liquid miscibility gap. The effect of changing the ratio of the molar volumes of the nonsolvent and the solvent is presented in Figure 5. Variation of $s$ results in minor differences in location of the miscibility gap. On the other hand, the influence of $s$

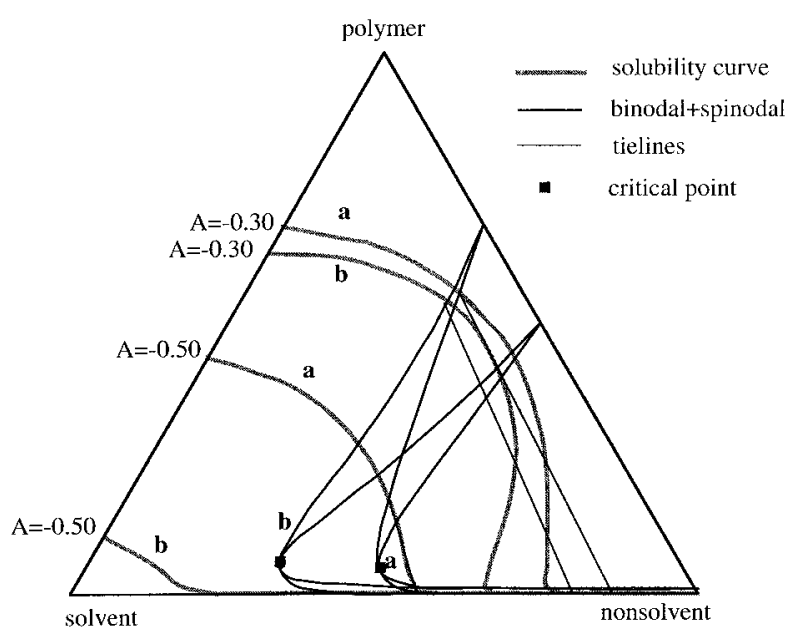

Figure 4. The influence of changes in $\chi_{23}$ on the location of the solubility curve and the binodal and the spinodal in the phase diagram. The transitions are presented for two different sets of interaction parameters. $s=1 ; r=0.004 ; \chi_{12}=0.5 ; \chi_{13}=1.0 ;$ a ) $\left.\chi_{23}=0.2 ; \mathrm{b}\right)$ $\chi_{23}=0.5$. 


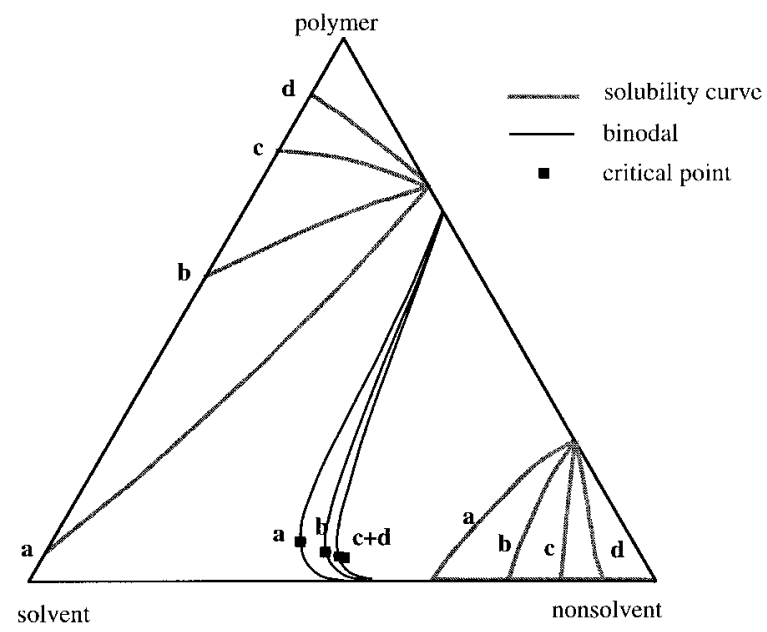

Figure 5. The effect of changes in $s$, the molar volume ratio of the solvent and the nonsolvent, on the phase diagram. $A=-0.20 . \chi_{12}=0.5 ; \chi_{13}=1 ; \chi_{23}=0.2 ; r$ $=0.004$. Values for $s$ for the different curves: a: $s$ $=0.25, \mathrm{~b}: s=0.5, \mathrm{c}: s=1, \mathrm{~d}: s=2$.

on the solubility curve is enormous. The molar volume of the nonsolvent is kept constant; therefore, the solubility curves always start at the same point on the polymer/nonsolvent axis. Increases in $s$ imply a decrease in the molar volume of the solvent. If the molar volume of the solvent is much larger than the molar volume of the nonsolvent the curve representing the solid-liquid transition increases steeply to higher polymer concentrations with increasing nonsolvent concentrations. The opposite effect is observed for small molar volumes of the solvent.

It is well known that the melting point depression of a polymer in a solvent depends strongly on the size of the solvent molecule. For example, the melting point depression of crystals in polymer blends is very small compared to the melting point depression in polymer solvents due to the lower gain in entropy. These effects are much larger than the effects due to the values of the interaction parameters.

It is also obvious that the presence of a liquidliquid miscibility gap is not a necessary condition for precipitation. Precipitation of the polymer from the solvent can also occur by crystallization during immersion of a polymer solution in another good solvent with a sufficiently high molecular weight.

Implications of the Competition Between SolidLiquid Demixing and Liquid-Liquid Demixing on the Membrane Morphology

Only a very small number of studies are available that focus on the role of solid-liquid demixing on the morphologies of membranes obtained by the immersion precipitation proces. For thermally induced phase separation the influence of the competition between solid-liquid demixing and liquid-liquid demixing on the membrane morphology has been studied in more detail. Therefore, the basic principles will initially be discussed for binary systems and will then be extended to ternary systems.

In Figure 6(A) the solid-liquid transition and the liquid-liquid transition are shown for a binary system and a ternary system. The binary phase diagram is similar to those published by Burghardt and Cahn. ${ }^{4,33}$ The phase separation processes that occur after passing a phase boundary strongly depend on which phase boundary is passed and how deep the miscibility gap is entered. Several cases can be distinguished.

In Figure 6(A) the cooling trajectories for polymer solutions with varying initial polymer concentrations are indicated. For the ternary solutions some composition paths are indicated in Figure 6(B) for a fixed composition of the casting solution. A composition path describes the timeand place-dependent composition of the polymer solution between immersion and phase separation. ${ }^{24}$ Notice that, in analogy with Figure 6(A), the polymer concentration can induce similar effects. In case 6 only the solid-liquid miscibility gap is entered and in case 3 only the liquid-liquid miscibility gap is entered. The structure generation under these conditions is well established.

Solid-liquid demixing processes can give rise to the formation of single lamellae at very low polymer concentrations or supramolecular assemblies of lamellae-like axialities and spherulites at high polymer concentrations. ${ }^{5-10,17,18,22}$ Solid-liquid demixing will occur at low nonsolvent concentrations and high polymer concentrations and also at very low polymer concentrations and high nonsolvent concentrations [not indicated in Fig. 6(B)].

For liquid-liquid demixing three types of phase separation mechanisms can be distinguished. ${ }^{11,34}$ At low polymer concentrations (between the binodal and the spinodal) phase separation takes place by nucleation and growth of a polymer-rich phase and polymer spheres can be obtained. At intermediate polymer concentrations spinodal demixing processes give rise to bicontinuous network structures. At high polymer concentrations (between the binodal and the spinodal) phase separation takes place by nucleation and growth of a polymer-poor phase and spongy structures can be obtained. If no other transition 


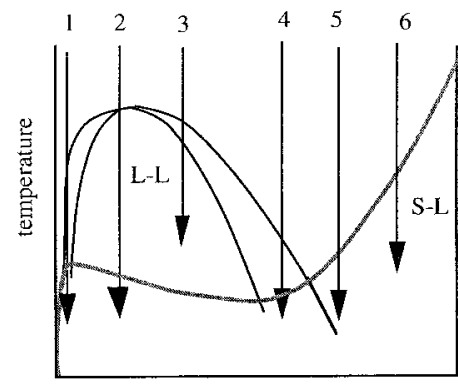

polymer concentration

A

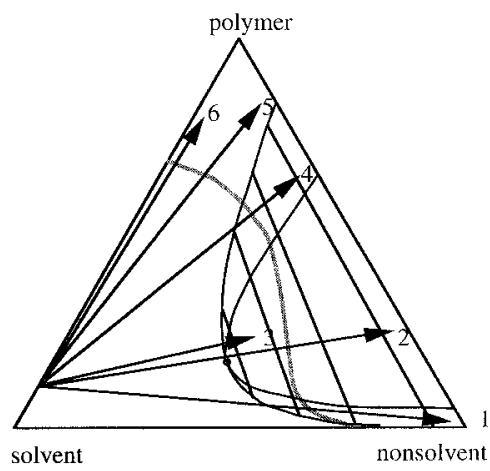

$\mathbf{B}$

Figure 6. Phase diagrams for binary systems and ternary systems. For arrows and numbers see text. (A) Nonequilibrium phase diagram for a binary system; (B) nonequilibrium phase diagram for a ternary system.

interferes with the liquid-liquid demixing process, coarsening phenomena driven by interfacial energy will induce complete phase separation in two layers. ${ }^{12,17,35}$

In cases 1,2 , and 4 crystallization will only take place after liquid-liquid demixing. Two reasons are responsible for this. ${ }^{33}$ Unstable solidliquid equilibria increase the free energy of mixing. For metastable solid-liquid equilibria the decrease in free energy of mixing for crystallization is smaller than for liquid-liquid demixing. In addition the activation energy for liquid-liquid demixing is much lower than for crystallization.

In case 1 first liquid-liquid demixing will take place by nucleation and growth of polymer-rich phase followed by crystallization of the polymerrich droplets. This case has been described for binary polymer-solvent combinations by Schaaf et al. and Berghmans et al. and for binary polymer blends by Shibanov et al. ${ }^{36-38}$ Schaaf et al. demonstrated that in case liquid-liquid demixing preceded solid-liquid demixing very smooth particles were obtained and for direct solid-liquid demixing rough particles. Shibanov et al. showed for polymer blends that the crystallization of particles rich in the crystallizable component was induced by neighboring particles resulting in a clear growth front. No accounts in literature have been found for ternary solutions.

In case 2 liquid-liquid demixing proceeds by spinodal decomposition. For binary solutions of isotactic polypropylene Lloyd et al. succeeded in obtaining bicontinuous structures stabilized by crystallization. ${ }^{5-7}$ For binary polymer blends the same was reported by Shibanov et al. and Inaba et al. ${ }^{38,39}$ Shibanov concluded that in contrast to the results of Inaba et al. and Lloyd et al. the spinodal morphology was significantly distorted by the crystallization process.

In case 4 the liquid-liquid demixing proceeds by nucleation and growth of a polymer-poor phase followed by crystallization of the matrix. This morphology has been obtained by ourselves for the immersion precipitation of poly-L-lactide and by Bulte et al. for nylon-4,6. ${ }^{17,22}$ Lloyd et al. and Aubert et al. obtained this morphology for binary solutions. ${ }^{5-8}$

In case 5 crystallization takes place first; however, in the course of the demixing process the polymer solution will be pushed in the miscibility gap. Examples of liquid-liquid demixing induced by solid-liquid demixing are presented for polymer blends by $\mathrm{Li}$ et al., and Tanaka and Nishi. ${ }^{39-43}$ These phenomena were recentely reported by van de Witte et al. for the immersion precipitation of poly-L-lactide. ${ }^{17}$ Spherulites surrounded with porous shells could be obtained. This morphology was attributed to a cascade of phase transitions. First solid-liquid demixing induced the formation of spherulites. Due to the spherulite growth liquidliquid was induced in the medium surrounding the spherulite. Crystallization occurred again in the polymer-rich phase generated during the liquidliquid demixing process.

\section{Parameters That Influence the Competition Between Liquid-Liquid Demixing and Solid-Liquid Demixing During Immersion Precipitation}

The relative position of the solubility curve with respect to the liquid-liquid miscibility gap is expected to play a large role in the phase separation 
of the solution. As has been demonstrated in detail in the theoretical section the most important variables on the relative position of the solid-liquid transition and the liquid-liquid transition are the driving force for crystallization of the polymer $(\mathrm{A})$, the nonsolvent/solvent molar ratio $(s)$, and the polymer-solvent interaction parameter $\left(\chi_{23}\right)$. More negative values for $A$ imply a higher tendency for crystallization. But also increases in $\chi_{23}$ and decreases in $s$ are expected to promote solidliquid demixing over liquid-liquid demixing. High polymer concentrations of the casting solution promote solid-liquid demixing because the composition is located closer to the solid-liquid miscibility gap.

Apart from thermodynamical parameters also kinetic parameters will play an important role. Liquid-liquid demixing usually proceeds very rapidly. The rate of crystallization of the polymer depends strongly on the properties of the polymer, the composition of the solution, and the conditions. Due to the higher activation energy necessary for crystallization and the lower growth rates of crystallites liquid-liquid demixing will usually precede solid-liquid demixing. ${ }^{18,33}$ Liquid-liquid demixing processes can even precede solid-liquid demixing processes in cases where solid-liquid demixing is favored thermodynamically. ${ }^{20}$

When the rate of crystallization of the polymer in solution is very slow compared to the quench rate the semicrystalline polymer will behave like an amorphous polymer. Low solvent/nonsolvent exchange rates-comparable to slow cooling in binary systems - will promote solid-liquid demixing processes that are favored thermodynamically. For the exchange rate it has been demonstrated that a good solvent-nonsolvent interaction, coupled with high mutual diffusion coefficients, will usually result in a very rapid exchange of solvent for nonsolvent. ${ }^{24,25}$ These conditions promote liquid-liquid demixing over solid-liquid demixing.

Some of these predictions are confirmed by examples from literature. The effect of the polymer/ solvent interaction parameter has been demonstrated for the immersion precipitation of poly-Llactide. ${ }^{17}$ If moderately concentrated solutions of poly-L-lactide in chloroform are immersed in methanol highly crystalline membranes are obtained with a cellular morphology. When a part of the chloroform in the casting solution is replaced by the poorer solvent toluene membranes are obtained with leafy structures due to crystallization. ${ }^{21}$ When chloroform is completely replaced by the poorer solvent dioxane the same effect is observed. ${ }^{45}$ Probably the lower solvent quality of the chloroform/toluene mixture and dioxane shifted the demixing mechanism from liquid-liquid demixing to solid-liquid demixing.

The effect of the polymer concentration in the casting solution is also clear from the system PLLA-chloroform-methanol. Crystalline membranes with cellular morphologies were obtained for casting solutions with low polymer concentrations. For casting solutions with high polymer concentrations spherulitic morphologies were obtained. ${ }^{44}$ Similar effects were observed for the system nylon-4,6-formic acid-water. ${ }^{22}$ An example of a membrane-forming system with a slowly crystallizing polymer is polyphenyleneoxide-trichloroethylene-ethanol. ${ }^{15}$ Thermodynamically, crystallization is favored over almost the entire composition range. However, the membranes that are obtained experimentally are amorphous. In these cases the morphology is determined by liquidliquid demixing followed by vitrification of the polymer-rich phase.

\section{CONCLUSIONS}

Many kinetic and thermodynamic parameters are important for the competition between solid-liquid demixing and liquid-liquid demixing during immersion precipitation. It is shown that nonequilibrium transitions can play a large role during the phase separation of polymer solutions and that the final morphology of the membrane can originate from a cascade of phase transitions. It is further demonstrated that, for a given polymer, the molar volume ratio of the solvent and the nonsolvent and the polymer-solvent interaction parameter can be used most efficiently to shift the importance from solid-liquid demixing to liquidliquid demixing and vice versa.

The authors would like to acknowledge the helpful discussions with Prof. R. Koningsveld.

\section{REFERENCES AND NOTES}

1. M. Mulder, Basic Principles of Membrane Technology, Kluwer Academic Publishers, Dordrecht, 1992.

2. H. K. Lonsdale, J. Membr. Sci., 10, 81 (1982).

3. F. C. Frank and A. Keller, Polym. Commun., 186, 29 (1988).

4. W. R. Burghardt, Macromolecules, 22, 2482 (1989). 
5. D. R. Lloyd, K. E. Kinzer, and H. S. Tseng, J. Membr. Sci., 52, 239 (1990).

6. S. S. Kim and D. R. Lloyd, J. Membr. Sci., 64, 13 (1991).

7. D. R. Lloyd, S. S. Kim, and K. E. Kinzer, J. Membr. Sci., 64, 1 (1991).

8. J. H. Aubert, Macromolecules, 21, 3468 (1988).

9. R. C. Domszy, R. Alamo, C. O. Edwards, and L. Mandelkern, Macromolecules, 19, 310 (1986).

10. L. Mandelkern, C. O. Edwards, R. C. Domszy, and M. W. Davidson, in Polymer Science and Technology Vol 30: Microdomains in Polymer Solutions, P. Dubin, Ed., Plenum Press, New York/London, 1985 , p. 121.

11. R. M. Hikmet, S. Callister, and A. Keller, Polymer, 29, 1378 (1988).

12. F-J. Tsai and J. M. Torkelson, Macromolecules, 23, 4983 (1990).

13. F. W. Altena, J. S. Schroder, R. Van de Huls, and C. A. Smolders, J. Polym. Sci. Part B: Polym. Phys., 24, 1725 (1986).

14. F. W. Altena and C. A. Smolders, Macromolecules, 15, 1491 ( 1982 ).

15. W. R. Burghardt, L. Yilmaz, and A. J. McHugh, Polymer, 28, 2085 (1987).

16. L. Yilmaz and A. J. McHugh, J. Appl. Polym. Sci., 31, 997 (1986).

17. P. van de Witte, H. Esselbrugge, P. J. Dijkstra, J. W. A. van den Berg, and J. Feijen, J. Membr. Sci., 113, 223 (1996).

18. P. van de Witte, P. J. Dijkstra, J. W. A. van den Berg, and J. Feijen, Polym. Prepr., 35, 842 (1994).

19. P. van de Witte, P. J. Dijkstra, J. W. A. van den Berg, and J. Feijen, to appear.

20. P. van de Witte, A. Boorsma, H. Esselbrugge, P. J. Dijkstra, J. W. A. van den Berg, and J. Feijen, Macromolecules, 29, 212 (1996).

21. P. van de Witte, H. Esselbrugge, A. M. P. Peter, P. J. Dijkstra, J. Feijen, R. J. J. Groenewegen, J. Smid, J. M. Olijslager, M. J. D. Eenink, and A. P. Sam, J. Controlled Release, 24, 61 (1993).

22. A. M. W. Bulte, B. Folkers, M. H. V. Mulder, and C. A. Smolders, J. Appl. Polym. Sci., 50, 13 (1993).
23. R. M. Boom, Th. van den Boomgaard, and C. A. Smolders, Macromolecules, 27, 2034 (1994).

24. A. J. Reuvers, J. W. A. van den Berg, and C. A. Smolders, J. Membrane Sci., 34, 45 (1987).

25. A. J. McHugh and C. S. Tsay, J. Appl. Polym. Sci., 46, 2011 (1992).

26. L. P. Cheng, A. H. Dwan, and C. C. Gryte, J. Polym. Sci. Part B: Polym. Phys., 32, 1183 (1994).

27. P. J. Flory, Principles of Polymer Chemistry, Cornell University Press, Ithaca, NY, 1953.

28. M. Kurata, Thermodynamics of Polymer Solutions, Harwood Academic, London, 1982.

29. H. Tompa, Polymer Solutions, Butterworths Scientific Publishers, London, 1956.

30. J. Pouchly, A. Zivny, and K. Solc, J. Polym. Sci. Part C, 23, 245 (1968).

31. J. Pouchly and A. Zivny, Makromol. Chem., 184, 2081 (1983).

32. R. Koningsveld, W. H. Stockmayer, and E. Nies, Makromol. Chem. Macromol. Symp., 39, 1 (1990).

33. J. W. Cahn, Trans. Metal. Soc. Aime, 242, 242 (1968).

34. K. Binder, in Materials Science and Technology, A Comprehensive Treatment Vol. 5. Phase Transformations in Materials, P. Haasen, Ed., VCH Publishers, New York, 1991, p. 405.

35. L. P. McMaster, Adv. Chem. Ser., 142, 43 (1975).

36. P. Schaaf, B. Lots, and J. C. Wittman, Polymer, 28, 193 (1987).

37. L. Aerts, M. Kunz, H. Berghmans, and R. Koningsveld, Makromol. Chem., 194, 2697 (1993).

38. Y. D. Shibanov and Y. K. Godovsky, Prog. Colloid Polym. Sci., 80, 110 (1989).

39. N. Inaba, K. Sato, S. Suzuki, and T. Hashimoto, Macromolecules, 19, 1690 (1986).

40. H. Tanaka and T. Nishi, Phys. Rev. Lett., 55, 1102 (1985).

41. H. Tanaka and T. Nishi, Phys. Rev. A, 39, 783 (1989).

42. Y. Li and B-J. Jungnickel, Colloid Polym. Sci., 269, 772 (1991).

43. Y. Li and B-J. Jungnickel, Polymer, 34, 9 (1993).

44. H. K. Lee, A. S. Myerson, and K. Levon, Macromolecules, 25, 4002 (1992).

45. P. van de Witte, P. J. Dijkstra, J. W. A. van den Berg, and J. Feijen, to appear. 